\title{
Solving large scale Max Cut problems via tabu search
}

\author{
Gary A. Kochenberger • Jin-Kao Hao • \\ Zhipeng Lü • Haibo Wang • Fred Glover
}

Received: 31 August 2010 / Accepted: 1 July 2011

(C) Springer Science+Business Media, LLC 2011

\begin{abstract}
In recent years many algorithms have been proposed in the literature for solving the Max-Cut problem. In this paper we report on the application of a new Tabu Search algorithm to large scale Max-cut test problems. Our method provides best known solutions for many well-known test problems of size up to 10,000 variables, although it is designed for the general unconstrained quadratic binary program (UBQP), and is not specialized in any way for the Max-Cut problem.
\end{abstract}

Keywords Max Cut problem · Metaheuristics $\cdot$ Combinatorial optimization

\section{Introduction}

Given an undirected graph $G(V, E)$ with edge weights $w_{i j}$, the NP-hard Max-Cut (MC) problem seeks a partition $S_{1} \subset V$ and $S_{2}=V-S_{1}$ such that the weight of the cut, defined as the sum of the weights on the edges connecting the two sets, is maximized. This problem has long served as a challenging test for researchers testing new

\author{
G.A. Kochenberger \\ University of Colorado, Denver, CO, USA \\ e-mail: gary.kochenberger@ucdenver.edu \\ J.-K. Hao · Z. Lü \\ LERIA, Universite d'Angers, 49045, Angers Cedex 01, France \\ J.-K. Hao \\ e-mail: hao@info.iniv-angers.fr \\ H. Wang \\ Texas A\&M International University, Laredo, TX, USA \\ e-mail: hwang@tamiu.edu \\ F. Glover $(\varangle)$ \\ OptTek Systems, Boulder, CO 80302, USA \\ e-mail: glover@opttek.com
}


methods for combinatorial algorithms. The problem also has well known practical applications in several areas including statistical physics, VLSI design, classification, and social network analysis. Other applications, as discussed by Boros and Hammer (1991), include fault test generation, multiprocessor assignments in distributed networks, image enhancement, and maximum likelihood rankings in statistics. Additional applications are discussed in the survey paper by Poljak and Tuza (1995).

The computational challenge of the Max-Cut problem has motivated a variety of solution approaches ranging from approximation algorithms based on semidefinite programming, to metaheuristic methods, to exact methods. The approximation algorithms, represented by the groundbreaking work of Goemans and Williamson (1995) and the later work by Karish et al. (2000) provide a performance guarantee but are generally outperformed by other methods in computational testing. Recent reports on exact methods include the cut and price approach by Krishnan and Mitchell (2006) and the branch and bound approach of Rendl et al. (2008). While these approaches represent advances over the approximation algorithms in finding optimal solutions to Max-Cut instances, their applications have proven to be limited to problems with no more than a few hundred nodes.

For larger MC instances, those with thousands of nodes, metaheuristic methods are required. In this regard, several advances have been recently reported in the literature, the most notable of which are the rank- 2 relaxation method, called CirCut, of Burer et al. (2001), the hybrid randomized method of Festa et al. (2002), and the scatter search method of Marti et al. (2009). Computational testing indicates that these methods generally outperform other methods in the literature with the scatter search method providing the best overall performance on test problems with up to 3000 nodes.

\section{Unconstrained binary quadratic programming and the Max-Cut problem}

Much of the published research on the Max-Cut problem present algorithms specially crafted for the problem at hand. Our approach taken here is quite different. In this paper we report on the application of a new tabu search method for the Unconstrained Binary Quadratic Program (UBQP) to the Max-Cut problem. Our approach is not specialized in any way for the Max-Cut problem but instead is designed for the general UBQP.

It is well known (see for example Boros and Hammer 1991; Helmberg and Rendl 1996) that the Max-Cut problem can be modeled as an integer program of the form:

$$
\begin{aligned}
& \max \frac{1}{2} \sum_{1 \leq i<j \leq n} w_{i j}\left(1-y_{i} y_{j}\right) \\
& \text { subject to: } y_{i} \in\{-1,1\} \quad \forall i \in V
\end{aligned}
$$

To solve this problem, we first introduce a change of variables $\left(y_{i}=2 x_{i}-1\right)$ leading to the unconstrained quadratic program in binary variables

$$
\max \sum_{i<j} w_{i j}\left(x_{i}+x_{j}-2 x_{i} x_{j}\right) ; \quad x_{j} \in\{0,1\}
$$


This problem is of the form max $x^{\prime} Q x$ and thus is solvable by the recently developed Diversification Driven Tabu Search (DDTS) method by Glover et al. (2010) for the general UBQP problem.

The DDTS method repeatedly alternates between a simple version of tabu search (TS) and a diversification phase founded on a memory-based perturbation operator. Starting from an initial random solution, DDTS uses the TS procedure to reach a local optimum. Then, the perturbation operator is applied to displace the solution to a new region, whereupon a new round of TS is launched. To facilitate achieving effective diversification, the perturbation operator is guided by information from a special memory structure.

This tabu search procedure uses a neighborhood defined by the single 1-flip moves, which consists of changing (flipping) the value of a single variable $x_{j}$ to its complement value $1-x_{j}$. The implementation of this neighborhood uses a fast incremental evaluation technique to calculate the cost of candidate moves. The diversification strategy utilizes a memory- based perturbation operator composed of three parts: a flip frequency memory, an elite solution memory, and an elite value frequency memory. These memory structures are used jointly by the perturbation operator to enhance the diversification of the search process. Complete details of this method are given in Glover et al. (2010).

\section{Computational results}

Due to the fact that the leading exact methods can only handle problems containing up to a few hundred binary variables, we restrict our comparisons to the best the literature has to offer coming from metaheuristic methods, which have demonstrated an ability to handle problems an order of magnitude larger.

Our computational testing was carried out on the " $G$ " problems available at http://www.stanford.edu/ yyye/yyye/Gset/. This set of test problem of medium to large size is widely adopted in the literature to facilitate computational testing and comparisons. In all, we considered 69 problems ranging in size from $n=800$ variables to $\mathrm{n}=10,000$ variables.

The recent paper of Marti et al. (2009) describes the use of a special purpose Scatter Search (SS) method for the Max-Cut problem and provides comparative results with widely regarded solution procedures such as GRASP Festa et al. (2002) and CirCut (Burer et al. 2001) on some of these same "G" problems. Their scatter search method, which we refer to by MDL, proved to outperform competing metaheuristic methods on most test problems considered and thus we use the MDL results as our main benchmark for making comparisons with our method. Our testing however, went beyond comparisons with MDL in terms of problem size, as results for the MDL method were reported only for problems up to $n=3000$. To provide a benchmark for comparison for larger problems (up to $n=10,000$ variables) we include published results from Choi and Ye (2000), which is the only published account we can find that reports on these larger instances.

Results from our testing are shown in Table 1 where the column titled CY refers to results published by Choi and Ye, the column titled MDL gives the results published 
Table 1 Computational results

\begin{tabular}{|c|c|c|c|c|c|c|}
\hline $\begin{array}{l}\text { Problem } \\
\text { ID }\end{array}$ & $\begin{array}{l}\# \\
\text { Vars }\end{array}$ & $\begin{array}{l}\text { Best known } \\
\text { OBJ }\end{array}$ & $\begin{array}{l}\text { SDP } \\
\text { bound }\end{array}$ & $\begin{array}{l}\text { CY } \\
\text { OBJ }\end{array}$ & $\begin{array}{l}\text { MDL } \\
\text { OBJ }\end{array}$ & $\begin{array}{l}\text { KHLWG } \\
\text { OBJ }\end{array}$ \\
\hline G1 & 800 & 11624 & 12078 & - & 11624 & 11624 \\
\hline $\mathrm{G} 2$ & 800 & 11620 & 12084 & - & 11620 & 11620 \\
\hline G3 & 800 & 11622 & 12077 & - & 11622 & 11620 \\
\hline G4 & 800 & 11646 & - & - & 11646 & 11646 \\
\hline G5 & 800 & 11631 & - & - & 11631 & 11631 \\
\hline G6 & 800 & 2178 & - & - & 2165 & 2178 \\
\hline G7 & 800 & 2003 & - & - & 1982 & 2006 \\
\hline G8 & 800 & 2003 & - & - & 1986 & 2005 \\
\hline G9 & 800 & 2048 & - & - & 2040 & 2054 \\
\hline G10 & 800 & 1994 & - & - & 1993 & 2000 \\
\hline G11 & 800 & 564 & 627 & 542 & 562 & 564 \\
\hline G12 & 800 & 556 & 621 & 540 & 552 & 556 \\
\hline G13 & 800 & 580 & 645 & 564 & 578 & 580 \\
\hline G14 & 800 & 3060 & 3187 & 2982 & 3060 & 3061 \\
\hline G15 & 800 & 3049 & 3169 & 2975 & 3049 & 3050 \\
\hline G16 & 800 & 3045 & 3172 & - & 3045 & 3052 \\
\hline G17 & 800 & 3043 & - & - & 3043 & 3046 \\
\hline G18 & 800 & 988 & - & - & 988 & 991 \\
\hline G19 & 800 & 903 & - & - & 903 & 904 \\
\hline G20 & 800 & 941 & - & 876 & 941 & 941 \\
\hline G21 & 800 & 931 & - & 855 & 930 & 931 \\
\hline G22 & 2000 & 13346 & 14123 & 12989 & 13346 & 13359 \\
\hline G23 & 2000 & 13317 & 14129 & 13006 & 13317 & 13342 \\
\hline $\mathrm{G} 24$ & 2000 & 13314 & 14131 & 12985 & 13303 & 13337 \\
\hline $\mathrm{G} 25$ & 2000 & 13326 & - & - & 13320 & 13332 \\
\hline G26 & 2000 & 13314 & - & - & 13294 & 13328 \\
\hline G27 & 2000 & 3318 & - & - & 3318 & 3336 \\
\hline G28 & 2000 & 3285 & - & - & 3285 & 3295 \\
\hline G29 & 2000 & 3389 & - & - & 3389 & 3391 \\
\hline G30 & 2000 & 3403 & - & 3080 & 3403 & 3403 \\
\hline G31 & 2000 & 3288 & - & 2936 & 3288 & 3288 \\
\hline G32 & 2000 & 1398 & 1560 & 1338 & 1398 & 1406 \\
\hline G33 & 2000 & 1376 & 1537 & 1330 & 1362 & 1378 \\
\hline G34 & 2000 & 1372 & 1541 & 1334 & 1364 & 1378 \\
\hline G35 & 2000 & 7670 & 8000 & - & 7668 & 7678 \\
\hline G36 & 2000 & 7660 & 7996 & - & 7660 & 7670 \\
\hline G37 & 2000 & 7666 & 8009 & - & 7664 & 7682 \\
\hline G38 & 2000 & 7681 & - & - & 7681 & 7683 \\
\hline G39 & 2000 & 2395 & - & - & 2393 & 2397 \\
\hline G40 & 2000 & 2387 & - & - & 2374 & 2390 \\
\hline
\end{tabular}


Table 1 (Continued)

\begin{tabular}{|c|c|c|c|c|c|c|}
\hline $\begin{array}{l}\text { Problem } \\
\text { ID }\end{array}$ & $\begin{array}{l}\# \\
\text { Vars }\end{array}$ & $\begin{array}{l}\text { Best known } \\
\text { OBJ }\end{array}$ & $\begin{array}{l}\text { SDP } \\
\text { bound }\end{array}$ & $\begin{array}{l}\mathrm{CY} \\
\mathrm{OBJ}\end{array}$ & $\begin{array}{l}\text { MDL } \\
\text { OBJ }\end{array}$ & $\begin{array}{l}\text { KHLWG } \\
\text { OBJ }\end{array}$ \\
\hline G41 & 2000 & 2398 & - & - & 2386 & 2400 \\
\hline G42 & 2000 & 2469 & - & - & 2457 & 2469 \\
\hline G43 & 1000 & 6659 & 7027 & - & 6656 & 6660 \\
\hline G44 & 1000 & 6648 & 7022 & - & 6648 & 6639 \\
\hline G45 & 1000 & 6652 & 7020 & - & 6642 & 6652 \\
\hline G46 & 1000 & 6645 & - & - & 6634 & 6649 \\
\hline G47 & 1000 & 6656 & - & - & 6649 & 6665 \\
\hline G48 & 3000 & 6000 & - & 6000 & 6000 & 6000 \\
\hline G49 & 3000 & 6000 & - & 6000 & 6000 & 6000 \\
\hline G50 & 3000 & 5880 & - & 5880 & 5880 & 5880 \\
\hline G51 & 1000 & 3846 & - & - & 3846 & 3847 \\
\hline G52 & 1000 & 3849 & - & - & 3849 & 3849 \\
\hline G53 & 1000 & 3846 & - & - & 3846 & 3848 \\
\hline G54 & 1000 & 3846 & - & - & 3846 & 3851 \\
\hline G55 & 5000 & 9960 & - & 9960 & - & 10236 \\
\hline G56 & 5000 & 3649 & - & 3649 & - & 3934 \\
\hline G57 & 5000 & 3220 & - & 3220 & - & 3460 \\
\hline G58 & 5000 & - & - & - & - & 19248 \\
\hline G59 & 5000 & - & - & - & - & 6019 \\
\hline G60 & 7000 & 13658 & - & 13658 & - & 14057 \\
\hline G61 & 7000 & 5273 & - & 5273 & - & 5680 \\
\hline G62 & 7000 & 4612 & - & 4612 & - & 4822 \\
\hline G63 & 7000 & 8059 & - & 8059 & - & 26963 \\
\hline G64 & 7000 & 7861 & - & 7861 & - & 8610 \\
\hline G65 & 8000 & 13286 & - & 13286 & - & 5518 \\
\hline G66 & 9000 & - & - & - & - & 6304 \\
\hline G67 & 10000 & - & - & - & - & 6894 \\
\hline G70 & 10000 & 9499 & - & 9499 & - & 9458 \\
\hline G72 & 10000 & 6644 & - & 6644 & - & 6922 \\
\hline
\end{tabular}

by Marti, Duarte and Laguna, and the right most column, titled KHLWG, gives our results. The Semi-Definite relaxation results have been reported in the literature for some of these problems and we list these in the forth column of the table to provide yet another benchmark for comparison. The third column in Table 1 gives the best known solutions reported in the literature prior to this paper. In Table 1 we report results from our method for each of the 69 problems under consideration. A "dash" in the table denotes that a result for that particular problem was not available. For example, MDL does not report results for problems G55 to G72.

To make an allowance for the different computers used by MDL and our DDTS algorithm, we used the standard SPEC benchmark data. The results reported by MDL 
were obtained by letting the method run for $1 / 2$ hour of CPU time on each problem. The comparable time on our computer, according to the SPEC 2000 and SPEC 2006 benchmarks is 2.36 hours of CPU time. Accordingly, the results we present in the table for problems G1-G54 refer to the best solution found by our method in 2.36 hours of CPU time. For the larger problems not addressed by MDL, we increased the CPU run time to allow our algorithm more time to search for good solutions as the problem size increased. Accordingly, for the $n=5000$ and 7000 variable problems, we report the best solutions found in 8 hours of CPU run time. For the 8,000, 9,000, and 10,000 variable problems, the CPU run time limits were 12 hours, 20 hours, and 24 hours respectively.

Table 1 enables a comparison of the DDTS metaheuristic with the scatter search metaheuristic of MDL on problems G1-G54. In a comparable time limit, our approach found better solutions on 40 of the 54 problems. On 12 problems both methods found the same solution and on 2 of the problems MDL found a better solution.

Over the entire test bed of 69 problems, Table 1 shows that our method matched best known solutions on 19 of the 69 problems, found new best known solutions on 46 problems, and failed to find best known solutions on 4 problems. On those problems for which a SDP bound is available, our objective function values are very close to the bounds, adding further evidence of the quality of our results.

\section{Summary \& conclusion}

We demonstrate that a modern tabu search metaheuristic designed for the general unconstrained binary quadratic program can produce high quality solutions to large Max-Cut problems. Comparisons with other approaches reported in the literature show that our method outperformed the leading competitive methods by a wide margin and in fact found new best known solutions for most problems attempted. This is particularly noteworthy in that the solution approach we employed is not specialized in any way for the Max-Cut problem, but handles problems of a much larger class. Our results suggest that further enhancements to solution methodologies for the unconstrained binary quadratic program hold considerable promise for solving even larger Max-Cut problems. We plan to report on such work in future papers.

\section{References}

Boros, E., Hammer, P.: The Max-Cut problem and quadratic 0-1 optimization; polyhedral aspects, relaxations and bounds. Ann. Oper. Res. 33, 151-180 (1991)

Burer, S., Monteiro, D., Zang, Y.: Rank-two relaxation heuristic for Max-Cut and other binary quadratic programs. SIAM J. Optim. 12, 503-521 (2001)

Choi, C., Ye, Y.: Solving sparse semidefinite programs using the dual scaling algorithm with an iterative solver. Working Paper, Department of Management Sciences, The University of Iowa (2000)

Festa, P., Pardalos, P., Resende, M., Ribeiro, C.: Randomized heuristics for the Max-Cut problem. Optim. Methods Softw. 7, 1033-1058 (2002)

Glover, F., Lü, Z., Hao, J.K.: Diversification-driven tabu search for unconstrained binary quadratic problems. 4OR, Q. J. Oper. Res. (2010). doi:10.1007/s10288-009-0115-y

Goemans, M., Williamson, D.: Improved approximation algorithms for Max-Cut and satisfiability problems using Semidefinite programming. J. ACM 42, 1115-1145 (1995) 
Helmberg, C., Rendl, F.: Solving quadratic (0-1) problems by semidefinite programs and cutting planes. Working Paper, Technische Universitat Graz (1996)

Karish, S., Rendl, F., Clausen, J.: Solving graph bisection problems with semidefinite programming. SIAM J. Comput. 12, 177-191 (2000)

Krishnan, K., Mitchell, J.: A semidefinite programming based polyhedral cut and price approach for the Max-Cut problem. Comput. Optim. Appl. 33, 51-71 (2006)

Marti, R., Duarte, A., Laguna, M.: Advanced scatter search for the Max-Cut problem. INFORMS J. Comput. 21, 26-38 (2009)

Rendl, F., Rinaldi, G., Wiegele, A.: Solving Max-Cut to optimality by intersecting semidefinite and polyhedral relaxations. Math. Program. 121, 307-335 (2008)

Poljak, S., Tuza, Z.: The Max-Cut problem: a survey. In: Cook, W., Lovasz, L., Seymour, P. (eds.) Special Year on Combinatorial Optimization. DIMACS Series in Discrete Mathematics and Computer Science. Am. Math. Soc., Providence (1995) 